\title{
POŠTA
}

TELEKOMUNIKÁCIE A

ELEKTRONICKY OBCHOD

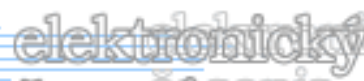

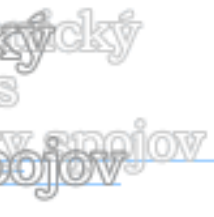

\section{PROBLEMATIK DER STUDIENGEBÜHREN - DARGESTELLT AM BEISPIEL DER BUNDESREPUBLIK DEUTSCHLAND}

\author{
Rudolf Brabeck ${ }^{1}$
}

\section{Brisanz des Themas}

Im Zeitalter der Globalisierung verstärkt sich die Intensität des Wettbewerbs zwischen Regionen und Ländern zwangsläufig auch im Ausbildungs- und Hochschulbereich. Vor diesem Hintergrund muss gesellschaftlich angestrebt werden, daß in unserer Informationsund Wissensgesellschaft tendenziell immer mehr junge Menschen eine möglichst hoch qualifizierte akademische Ausbildung absolvieren.

Die Diskussion über Studiengebühren staatlicher Hochschulen konzentriert sich, vereinfacht ausgedrückt, auf zwei Kernaspekte. Einerseits soll das Instrument der Studiengebühren die Effektivität der Hochschulausbildung verstärken, insbesondere sollen dadurch die Verkürzung der in Deutschland überdurchschnittlich langen Studiendauer und der Wettbewerb zwischen Hochschulen ausgelöst werden. Andererseits kann die Einführung eines Modells nicht ausgewogener Studiengebühren $\mathrm{zu}$ erheblichen sozialen Unverträglichkeiten führen: nicht die soziale Herkunft darf eine gewichtige Rolle spielen, sondern die berufliche Zukunft. Bildungspolitik ist schließlich auch vorbeugende Sozialpolitik. Geld, das für Ausbildung ausgegeben wird, zahlt sich in der Regel später um ein Vielfaches aus - die Arbeitslosenquote von Akademikern lag innerhalb des letzten Jahrzehnts immer unter 5 \% (Friedmann - 2005b - S. 70-80).

Die Grundüberlegung der Einführung von Studiengebühren geht davon aus, daß diejenigen zur nachgelagerten Finanzierung der Hochschulausbildung herangezogen werden, die mit größerer Wahrscheinlichkeit zukünftig zu den Besserverdienenden gehören werden, zudem verfügen Akademiker über ein großes soziales Ansehen. Die Rückzahlung soll nicht während des Studiums erfolgen.

\section{Voraussetzungen der Einführung von Studiengebühren}

2.1. Rechtliche Grundlagen: Urteil des Bundesverfassungsgerichts

Das Hochschulrahmengesetz (HRG) schloss bislang Studiengebühren aus. Angesichts des Ziels der damaligen rot-grünen Bundesregierung, gleichwertige Lebensverhältnisse in Deutschland zu schaffen, wurde diese gesellschaftliche Normvorstellung vor drei Jahren im HRG verankert: „Das Studium bis zum ersten berufsqualifizierenden Abschluss ist studiengebührenfrei.“ (Friedmann - 2005a - o.S). Dagegen klagten sechs Bundesländer. Das Bundesverfassungsgericht gab am 26. Januar 2005 den Ländern die Gesetzgebungskompetenz im Kultusbereich zurück, frei ist insbesondere der Weg für einen Feldversuch, in dem die Erwartungen und Befürchtungen, die sich mit der Einführung von Studiengebühren seit

\footnotetext{
${ }^{1}$ Diplom-Kaufmann Rudolf Brabeck, Fakultät für Management und Ökonomie des Transports und der Kommunikation, Universität Žilina, Rudolf@Brabeck.de
} 
langem verbinden, erfasst werden sollen (HoF - 2005 - S. 4). Das Bundesverfassungsgericht hat ausdrücklich darauf hingewiesen, dass sein Urteil keine Stellungnahme zur Sozialverträglichkeit verkörpert und dass die Richter keine Bildungspolitiker seien.

\subsection{Die Vereinbarung von Bologna}

Zwischenzeitlich haben 45 Staaten den Beschluss akzeptiert, die Internationalisierung im Bildungsbereich zu verstärken. Ein zentraler Gesichtspunkt ist, durch einheitliche oder vergleichbare Abschlüsse, gegenseitige Anerkennung und die Modularisierung von Ausbildungsinhalten die Studierquote (die in Deutschland unterdurchschnittlich ausgeprägt ist) zu erhöhen. Bis Ende des Jahrzehnts soll in Deutschland insbesondere das zweistufige System der jeweils berufsqualifizierenden Bachelor- und Master-Abschlüsse eingeführt werden. Zahlreiche deutsche Fakultäten und Bildungspolitiker äußern Kritik, weil eine durch die Einführung des Bachelor-Studiums mögliche - Verkürzung des Studiums das Qualitätsniveau sinken lassen kann (O.V.-1 - 2005 - o.S.).

\subsection{Aktuelle Finanzierungsengpässe des Bundes und der Länder}

Die Finanzsituation deutscher Hochschulen ist seit langem desolat. Die Mittelausstattung ist innerhalb des abgelaufenen Jahrzehnts nicht aufgestockt worden, obwohl die Anforderungen an technische Ausstattungen und die Zahl der Studenten kräftig angewachsen sind. In jüngerer Vergangenheit ist sogar ein Rückgang der finanziellen Mittel festzustellen gewesen, der sich trotz der weiterhin wachsenden Studentenzahlen fortsetzen wird. Gegenwärtig schultern die Länder rund $€ 20$ Mrd. und der Bund rund $€$ 2,5 Mrd. (Rudolf - 2004 - S. 2-3). Derzeit fehlen den deutschen Hochschulen rund € 3 Mrd. pro Jahr (Spiewak - 2005a - o.S.), und zusätzliches Geld kann nicht erwartet werden (Burtscheid $2005-0 . S$.$) .$

Im internationalen Vergleich gilt Deutschland schon lange als eines der Schlusslichter im Hinblick auf Bildungsausgaben. Dies ist daran abzulesen, daß hier nur 1,0 \% des Bruttoinlandsprodukts in dieses Zukunftsfeld investiert wird, diese Größenordnung ist etwa vergleichbar mit den USA, die nur 1,1 \% des Bruttoinlandsprodukts für Bildung aufbringen (Hartung - $2003-$ o.S.).

\section{Grundfragen}

Bislang ist die Rolle des Staates nicht hinreichend bestimmt, der in der Vergangenheit die Grundsicherung im schulischen und akademischen Bildungsbereich garantierte. Einerseits erhebt sich die Frage, warum nicht-akademische Qualifizierung - beispielsweise zum Physiotherapeuten oder zum Handwerksmeister - von den Auszubildenden, aber akademische Ausbildung von der öffentlichen Hand zu finanzieren ist (im Rahmen einer Einführung von Studiengebühren müßte dann auch die Ausbildungsförderung grundsätzlich reformiert werden). Andererseits: hängt das geringe Ausmaß bildungsferner Schichten an der Hochschulausbildung nicht erheblich stärker von der frühzeitigen Selektion im Schulsystem als von der Einführung oder der Höhe von Studiengebühren ab?

\section{Befürworter und Kritiker von Studiengebühren}

\subsection{Pro-Argumente}

Die gewichtigen Argumente für die Einführung von Studiengebühren lassen sich folgenden (selbst gebildeten) Kategorien zuordnen: sozialpolitische Aspekte, Vorteile für die Studierenden sowie Wirtschaftlichkeits- und Leistungsverbesserung für die Hochschulen. 


\subsubsection{Sozialpolitische Aspekte}

Das Ziel der sozialen Gerechtigkeit - mit der Konsequenz der Kostenbeteiligung bei der höheren Ausbildung - fungiert als Kernargument. Die Nachfrager nach Bildungsgut haben ihre Inanspruchnahme zu bezahlen, die Finanzierung der Hochschulausbildung „von unten nach oben“ muss beendet werden: nur etwa jedes 10. Kind, dessen Vater der niedrigen sozialen Herkunftsgruppe zugeordnet wird, nimmt ein Hochschulstudium auf, während sich 81 \% der Kinder aus hoher sozialer Herkunftsgruppe für ein Hochschulstudium entscheiden“ (Rudolf - 2004 - S. 14). Flankierend wird argumentiert, daß in Ländern, die Studiengebühren fordern, der Anteil immatrikulierter Arbeiterkinder höher als in Deutschland ist. In diesem Zusammenhang wird auch darauf hingewiesen, daß die jüngste OECD-Studie den empirisch belegten Nachweis zu erbringen glaubt, daß die Chancengleichheit im Bildungsbereich durch Studiengebühren nicht berührt wird (Etzold - 2005 - o.S.).

\subsubsection{Vorteile für die Studierenden}

Bildungsgut zu privatisieren, dies versetzt die Studentenschaft in die Position des Bildungskunden. Durch Studiengebühren, die ausschließlich den Hochschulen zufließen, werden sowohl die Ausbildungsqualität spürbar erhöht als auch die Studienbedingungen verbessert. Studiengebühren ermöglichen somit ein eigenverantwortlicheres, hochwertigeres und zielstrebigeres Studium.

\subsubsection{Wirtschaftlichkeits- und Leistungsverbesserung für die Hochschulen}

Angesichts der enormen finanziellen Herausforderungen des Bundes und der Länder können zusätzliche Mittel das Niveau in Forschung und Lehre spürbar anheben und den auch international nötigen - qualitativen Wettbewerb zwischen den Hochschulen entfachen. Zusätzlich werden Studiengebühren eine wichtige Steuerungsfunktion übernehmen: Abschlüsse von Studiengängen, die auf dem Arbeitsmarkt gegenwärtig nur geringe Bedeutung haben, werden zukünftig zwangsläufig nur noch in sehr geringem Maße nachgefragt (sog. Orchideenfächer). Insbesondere wird die Einführung von Studiengebühren damit begründet, daß renommierte ausländische Hochschulen ebenfalls Studiengebühren erheben.

\subsubsection{Zwingende Forderungen bei der Erhebung von Studiengebühren}

Aus der Sicht von Hochschulen ist als Voraussetzung zwingend, daß Studiengebühren autonom verwaltet werden dürfen, um die Qualität der Lehre (vor allem in Gestalt der Betreuungsrelation zwischen Dozenten und Studenten) verbessern und höhere Betreuungsstandards (insbesondere im Hinblick auf die Studienberatung) realisieren zu können.

Die Selbständigkeit der Hochschulen muß auch das Recht umfassen, innerhalb vorgegebener Bandbreiten die Höhe der Studiengebühren festzulegen und nach Studiengängen zu differenzieren.

Trotz der Bildungshoheit der Länder ist eine bundeseinheitliche Regelung erforderlich, vor allem im Hinblick auf die zulassungsbeschränkten Studiengänge, deren Studienplätze zentral zugeteilt werden (numerus clausus-Fächer).

\subsection{Contra-Argumente}

Die Verfechter einer Hochschulausbildung ohne Studiengebühren weisen mit Nachdruck darauf hin, daß in der globalen Wissensgesellschaft Bildung und Wissen die wichtigsten Rohstoffe darstellen. Wenn wir demzufolge eine höhere Akademikerquote anstreben müssen, dann ist dies im großen Interesse unserer Gesellschaft, die entsprechend die Finanzierung eines möglichst hohen Bildungsstandards zu übernehmen hat. Bildung wird zudem als menschliches Grundrecht gewertet: sie ist der Hebel, um die Welt zu verstehen. 
Die zahlreichen Argumente gegen die Einführung von Studiengebühren an öffentlichen Hochschulen können den - ebenfalls selbst bestimmten - Bereichen Studiengebühren als Abschreckung, mögliche Folgerisiken und Überforderung der Hochschulen zugeordnet werden.

\subsubsection{Studiengebühren als Abschreckung}

Die Erfahrungen in Österreich (dort werden zwischenzeitlich $€ \quad 360,00$ Studiengebühren pro Semester erhoben) zeigen augenfällig, daß seit der Einführung von Studiengebühren die Ersteinschreibungen massiv abnahmen und seitdem auch nicht mehr gestiegen sind (O.V.-3 - 2005 - o.S.). Zudem sollten Eltern von studierenden Kindern keine weiteren Belastungen entstehen. Eine zusätzliche psychologische Barriere zum Studium kann aufgebaut werden durch die begründbare Angst vor Verschuldung. Weil viele Studenten Nebenjobs anzunehmen gezwungen sind, wird ein zügiges Studieren erschwert (wer jobben muß, studiert länger). Und aus Gründen der Verschuldung wird die Familiengründung von Absolventen verschoben.

\subsubsection{Mögliche Folgerisiken}

Das Finanzvolumen für Hochschulen wird zukünftig reduziert werden, deshalb ist die Gefahr groß, daß die Einführung von Studiengebühren nicht zu qualitativen Verbesserungen führt, sondern nur eine Ausgleichsfunktion übernimmt. Im Gutachten von Professor Kirchhof wird zudem auf die rechtlich bedenkliche Situation hingewiesen, daß der Zugriff der Finanzminister auf die Studiengebühren nicht zu verhindern sei (Gräber - 2005 - o.S.).

Bei Studiengebühren in Höhe von $€ 500$ je Semester würden durchschnittlich nur 13 \% der Studienkosten abgedeckt (Müller-Böling - 2005 - o.S.). Hinzu kommt, daß die Einnahmen aus Studiengebühren durch hohe Verwaltungskosten geschmälert werden. Der Berliner Finanzsenator Sarrazin ist überzeugt, daß ,es sicher nicht bei $€ 500$ bleiben wird und daß die Gebühreneinnahmen nicht vollständig den Hochschulen zufließen müssten“ (O.V.-2 2005 - o.S.). Auch die Hochschul-Rektoren-Konferenz (HRK) meint, daß nur anfänglich einheitliche Studiengebühren in Höhe von $€ 500$ zu erwarten seien, „danach entscheiden die Hochschulen selbständig“ (O.V.-4 - 2005 - o.S.).

\subsection{3. Überforderung der Hochschulen}

Im Anschluß an das Urteil des Bundesverfassungsgerichts vom 26. Januar 2005 verkündeten mehrere Bundesländer, Studiengebühren ab 2006 zu fordern, aber bis heute hat kein einziges Bundesland ein Konzept dazu präsentiert. Die Übernahme der Inkasso-Funktion würde in vielen Hochschulen eine große, zusätzliche Verwaltungsaufgabe auslösen. Insbesondere erhebt sich die Frage, welcher Träger die Ausfallbürgschaft bei kreditfinanzierten Studiengebühren übernimmt, nachdem die Kreditanstalt für Wiederaufbau (KfW) die Wahrscheinlichkeit des Kreditausfalls auf 13 \% schätzt (Martens et al. - 2005 o.S.).

\section{Modelle zur Studienfinanzierung}

5.1. Darstellung des australischen Gebührenkonzepts als Bezugs-Modell

Das 1989 umgesetzte Higher Education Contribution System (HECS) gilt international als Grundmodell für Studiengebühren im Hochschulbereich. Gefördert wird durch Kredite und andere Maßnahmen bei gering verdienenden Eltern, ein staatliches Stipendium kommt hinzu. Die aufgelaufenen Studiengebühren (von jährlich rund $€ 2.000$ bis ca. $€ 3.500$ ) werden 
ab einem jährlichen Mindesteinkommen von ca. $€ 14.600$ durch Rückzahlung von 3 bis $6 \%$ des Einkommens getilgt (O.V.-5 - 2005 - o.S.)

Häufig wird in Deutschland das australische Modell als positives Beispiel gepriesen. Erwähnenswert ist in diesem Zusammenhang, daß in Australien die jährlichen Zuschüsse für Hochschulen Schritt für Schritt gesenkt und deshalb die Studiengebühren kontinuierlich angehoben werden (Spiewak - 2005b - o.S.).

\subsection{Im Ausland praktizierte Modelle von Studiengebühren staatlicher Hochschulen}

Eine standardisierte Einteilung von Studiengebühren pro Jahr wird bis dato nicht vorgenommen. Es können aber nach Plausibilität beispielsweise unterschieden werden (O.V-5 $-2005-0 . S$.):

a) Länder ohne Studiengebühren: Dänemark, Finnland und Schweden.

b) Länder mit moderaten Studiengebühren (i.d.R. bis € 1.000): Österreich, Portugal, Spanien und Frankreich.

c) Länder mit hohen Studiengebühren (i.d.R. bis € 2.500): Großbritannien, Italien, Kanada und Niederlande.

d) Länder mit sehr hohen Studiengebühren (i.d.R. ab € 3.500): Japan und USA.

\subsection{Bislang in Deutschland präsentierte Vorstellungen}

Die Anzahl der vorgestellten Modelle ist unübersehbar. Gemeinsam ist ihnen, daß sie tendenziell gegensätzliche Ziele zu erreichen trachten: den Hochschulen wollen sie durch Studiengebühren beträchtliche Einnahmen bescheren und zukünftige Akademiker nicht vom Studium abschrecken.

Quasi alle deutschen Bundesländer nutzen bislang nur Gebührenmodelle bei LangzeitStudenten. Das Einstiegsmodell mehrerer Bundesländer sah vor, Studiengebühren pro Jahr bei ca. $€ 1.000$ anzusiedeln, wobei beabsichtigt war, günstige Kredite über den Bankenmarkt zur Verfügung zu stellen und nach dem Start ins Berufsleben $8 \%$ vom Bruttogehalt der Absolventen zurückzuverlangen (O.V.-6 - 2005 - S. 2).

Erst später sollten die Hochschulen bis zu einer noch zu definierenden Obergrenze Studiengebühren autonom festlegen können.

Konkrete Modelle zur Finanzierung von Studiengebühren sind aber bis dato nicht beschlossen. Zu erwarten ist, dass die Kreditanstalt für Wiederaufbau (KfW) Darlehen anbieten wird, die auch zur Finanzierung von Lebenshaltungskosten abrufbar sind. Die KfW rechnet für ein Universitätsstudium mit durchschnittlichen Verbindlichkeiten (Studiengebühren und Lebenshaltungskosten) in Höhe von rund $€ 30.000$ (O.V.-11 - 2005 o.S.). Das KfW-Modell sieht vor: jeder Student bekommt, unabhängig vom Studienfach, grundsätzlich $€ 650$ pro Monat, maximal zehn Semester lang, der Zins liegt zwischen 5 und 5,5 \% (Gräber - 2005 - o.S.). Zwischenzeitlich bieten auch zahlreiche Banken Studenten Kredite an, wohl in erster Linie, um später wohlhabende Akademiker möglichst früh zu binden.

Das führende Bundesland bei der Einführung von Studiengebühren, BadenWürttemberg, rechnet mit $€ 150$ Millionen, wenn ab Wintersemester-2007/2008 Gebühren von $€ 500$ pro Studiensemester erhoben werden. Parallel dazu sollen Kredite landeseigener Banken zur Verfügung gestellt werden, Details sind jedoch noch nicht bekannt (o.V.-9 - 2005 - S. 1).

Eine der führenden Institutionen im Bereich der Hochschulberatung - das Centrum für Hochschulentwicklung - plädiert in zahlreichen Publikationen für eine konsequente Wettbewerbsausrichtung: Hochschulen sollten nicht um öffentliche Gelder, sondern um die besten Studenten werben. 


\section{Studiengebühren aus der Sicht betroffener Personenkreise}

Die große Mehrheit der studentischen Vertretungen und der Gewerkschaften (verdi 2005 - S. 3-4) warnt seit langem vor der Einführung allgemeiner Studiengebühren, lediglich der Ring Christlich-Demokratischer Studenten (RCDS) plädiert für „,neue Wege der Hochschulfinanzierung“ (Einführung von Studiengebühren), um Hochschulen attraktiver zu machen (HoF - 2005 - S. 39). Es gab bereits flächendeckenden Protest, insbesondere in Baden-Württemberg und Nordrhein-Westfalen, aber noch keine Eskalation (Himmelrath 2005 - o.S.). In Köln waren 50.000 Studenten zur größten Protestaktion gegen die Einführung von Studiengebühren aufgerufen, aber nur 300 sind zur Vollversammlung erschienen (Hickmann et al. - 2005 - S. 13).

Im Gegensatz zu den Gewerkschaften, zum Studentenwerk und Studentenvertretungen haben der Präsident der Hochschulrektorenkonferenz und der Präsident der Deutschen Forschungsgemeinschaft erklärt, ein Verbot von Studiengebühren würde dem erklärten Ziel widersprechen, „Autonomie und Qualität der Hochschulen in Deutschland zu stärken...“ (O.V.-7 - 2005 - o.S.). Nach ihrer Ansicht wollen sie selbst darüber entscheiden, ob und für welche Studiengänge sie Gebühren und in welcher Abstufungsform einführen wollen. Die Hochschulen haben sich zur Sozialverträglichkeit von Gebührenmodellen verpflichtet, und sie wollen über die Verwendung selbst entscheiden, auch der Deutsche Hochschulverband (der Universitätsprofessoren) ist nicht gegen Studiengebühren eingestellt (CHE - 2001 - S. 2-6)

Der Stifterverband der Deutschen Wirtschaft gehört mit der KfW-Bankengruppe zu den expliziten Befürwortern von Studiengebühren, die Wirtschafts- und Arbeitgeberverbände sowie der Bundesverband der Deutschen Industrie sehen in Studiengebühren den Weg zu einer umfassenden Modernisierung von Hochschulen (HoF - 2005 - S. 40-43). Und Handelskammerpräsident Braun erklärte: Studiengebühren seien überfällig (O.V.-10 - 2005 o.S.)

Die CDU/CSU-Fraktion befand die Karlsruher Entscheidung „gut für unser Land“, auch die FDP begrüßte dieses Urteil, hingegen die SPD bezeichnete die Aufhebung des Verbots von Studiengebühren als „keinen guten Tag“ für die deutschen Studierenden und präferiert überwiegend das Modell der Studienkonten mit Langzeit-Studiengebühren, Bündnis 90/Die Grünen kommentierten den Karlsruher Spruch moderat und stellten fest, politisches Ziel müsse es sein, immer mehr junge Menschen zum Studium zu befähigen (O.V.-8 - 2005 o.S.), aber es wird ergänzt, daß ein gebührenfreies Erststudium nach wie vor die beste Voraussetzung bietet (HoF - $2005-$ S. 35).

\section{7. $\quad$ Entwicklung eines Studiengebühren-Modells}

Aus Gründen der Chancengerechtigkeit im Bereich der staatlichen Hochschulausbildung sollte das Erststudium - nicht nur das Bachelorstudium (B.S.), sondern auch das Masterstudium (M.S.) - generell kostenfrei sein, sofern der Student bei Studienbeginn nicht älter als 40 Jahre ist. Das BaföG muß parallel als dauerhaft sichere Lebens- und „Studier“grundlage erhalten bleiben.

Wird im Rahmen des Erststudiums die Regelstudienzeit im Grundstudium (analog: B.S.) um mehr als zwei Semester (Überziehungssemester) und / oder im Hauptstudium (analog M.S.) um mehr als zwei Semester (Überziehungssemester) überschritten, so sind diese Überziehungssemester kostenpflichtig.

Jedes Semester eines Zweistudiums (nach einem erfolgreich abgeschlossenen Erststudium) sollte grundsätzlich kostenpflichtig sein in Höhe der Selbstkosten (Differenzierung der Semesterkosten nach Studiengang). Wird ein Zweitstudium nach einem abgebrochenen Erststudium aufgenommen und erfolgreich abgeschlossen, dann sind nur die tatsächlich absolvierten Studiensemester des Erststudiums (eingeschriebene Semester) zu bezahlen, höchstens aber $€ 500$ pro Semester. 
Die Zahlung der Studiengebühren - für das überzogene oder abgebrochene Erststudium oder das Zweitstudium nach erfolgreichem Erststudium - beginnt drei Jahre nach Abschluß der Hochschulausbildung und beträgt 10 \% des Einkommens. Der Anspruch jedes

Bundeslandes auf Rückzahlung dieser Gelder, die in vollem Umfang an die jeweiligen Hochschulen fließen sollten, verjährt nicht. Maximal 60 \% der fälligen Studiengebühren können durch praktische Sozialarbeit in einschlägigen Einrichtungen getilgt werden.

Ausländische Studenten sind von der Zahlung von Studiengebühren grundsätzlich befreit, wenn in ihrem Heimatland Kostenfreiheit der Hochschulausbildung besteht, ansonsten haben sie die Selbstkosten zu finanzieren, maximal $€ 500$ pro Semester.

Die vorgeschlagenen Regelungen sollten bundeseinheitlich umgesetzt werden.

\section{8. $\quad$ Fazit}

Die Problematik im Rahmen der Diskussion über das Thema Studiengebühren läßt sich auf folgende Frageformel bringen: Effizienzgewinne oder Gefährdung der Chancengleichheit?

Zwischenzeitlich ist absehbar, daß Studiengebühren an öffentlichen deutschen Hochschulen - wahrscheinlich Ende 2007 - eingeführt werden, die genauen Modalitäten sind aber noch nicht bekannt. Wer sowohl einen gerechteren Hochschulzugang als auch eine höhere Akademikerquote anstrebt, muß ein Konzept zur Erhebung von Studiengebühren entwickeln, das weitgehend sozialverträglich ausgestaltet ist und ein stimmiges PreisLeistungs-Verhältnis der Studentenschaft bietet, um die Bereitschaft anzuheben, einen Teil der Ausbildungskosten selbst zu tragen.

Die Argumentation, daß Studiengebühren die Misere der Hochschulen - insbesondere durch die damit verbundene Initiierung des Wettbewerbs - entscheidend lindern, erscheint sehr verkürzt. Vor allem die mangelnde Autonomie der Hochschulen (besonders bezüglich der Finanzen) entpuppt sich als Kernproblem auf dem Wege zu einem effizienten Hochschulmanagement (ministerielle Vorgaben bei der Entwicklung, Profilierung und Schließung von Studiengängen, Vetorecht bei Berufungen etc. sind geradezu typisch). Der stärkste Effekt, um Wettbewerb zu schaffen, dürfte weniger von Studiengebühren, sondern hauptsächlich vom Recht der Hochschulen ausgehen, Eingangsprüfungen und Auswahlgespräche selbständig durchführen zu können. (Finetti - 2005 - S. 4). Bedrückend erscheint zudem, daß keine Sicherheit besteht, Studiengebühren dem Zugriff des Finanzministers zu entziehen.

Sollte der anzustrebende Wettbewerb zwischen Hochschulen eine nennenswerte Intensität annehmen, so würden rasch einige Elite-Hochschulen entstehen. Dieser Selektionsprozess würde dann den Großteil der Hochschulen zu Verlierern machen. Dies heißt: nicht Fähigkeiten, Wissen sowie Begabungen entscheiden letztlich über den Hochschulzugang, sondern die soziale Herkunft in Gestalt hoher Einkommen und wirtschaftlicher Stärke der Eltern.

Die aufgeführten skandinavischen Länder geben der Bildung absolute Priorität. Investitionen in das steigende Qualifikationsniveau gelten dort als gesellschaftlich wichtigste Aufgabe. Deshalb haben sich diese Länder für ein völlig kostenfreies Studium entschieden. Diese Argumentation ist humanistisch, sozial aufrichtig, materiell sehr überzeugend und letztlich auch an Erfolg nicht zu übertreffen (die skandinavischen Länder gelten als große Gewinner der sog. Pisa-Studien). 
Deshalb fußen die Eckpfeiler des kostenfreien Studiengebühren-Modells der Verfasserin auf dem Kerngedanken der skandinavischen Konzeption der Hochschulausbildung ohne Studiengebühren.

\section{Literaturverzeichnis}

[1] Burtscheid, Christine, Bayern: Universitäten wollen Studiengebühren sofort, in: sueddeutsche.de, 18.05.2005, www.sueddeutsche.de/,jkl1/jobkarriere/berufstudium/artikel/320/53267/

[2] Centrum für Hochschulentwicklung (CHE), Studiengebühren als Option für autonome Hochschulen, Gütersloh 2001

[3] Etzold, Sabine.:Studiengebühren, in: Die Zeit, Hochschule, 40/2001, www.zeit,de/archiv/2001/40/200140_mythos.xml, 13.02.2005

[4] Finetti, Marco.: Wer was studieren darf, in: Süddeutsche Zeitung, Meinung, Nr. 206, 07.09.2005

[5] Friedmann, Jan (a), Karlsruher Urteil - Gebührende Klarheit, in: Spiegel Online, 26. Januar 2005, www.spiegel.de/unispiegel/geld/0,1518,druck-338599,00.html, 13.02.2005

[6] Friedmann, Jan (b), Politik im Blindflug, in: Der Spiegel, Serie, 34/2005, Hamburg 2005

[7] Fritz-Vannahme, Joachim.: Gebühren für den Geist: Peter Glotz fordert Studiengebühren, in: Die Zeit, Wissen, 14/1996, www.zeit.de/archiv/1996/14/glotz.txt.19960329.xml, 13.02.2005

[8] Gräber, Berrit.: Aufgepasst beim Studium auf Pump, in: sueddeutsche de, 04.08.2005, www.sueddeutsche.de/,jkm3/jobkarriere/berufstudium/artikel/69/58011/, 04.08.2005

[9] Hartung, Manuel: Erst lernen, dann zahlen, in: Die Zeit, Chancen, 21/2003, www.zeus.de/text/2003/21/C-Studiengeb_9fhren2, 13.02.2005

[10] Hickmann, Christoph, und Finetti, Marco.: Warten auf den Zahltag, in: Süddeutsche Zeitung, Wissen, Nr. 121, 30.052005, Seite 13

[11] Himmelrath, Armin.: Studiengebühren: Protest in Südwest, in Spiegel Online, 03.05.2005,www.spiegel.de/unispiegel/geld/0,1518,druck-354501,00html, 04.05.2005

[12] HoF (Hrsg.), Institut für Hochschulforschung an der Martin-Luther-Universität HalleWittenberg, Studiengebühren nach dem Urteil, Arbeitsbericht-5-05, Wittenberg 2005

[13] Martens, Heike, und Neumann, Conny.: Hochschulen: Riskante Kredite, in Spiegel Online, 05.02.2005, www.spiegel.de/unispiegel/geld/0,1518,druck-340161.html, 13.02.2005

[14] Müller-Böling, Detlef Müller-Böling, Detlef.: Vorsicht, Mythenbildung, in: Die Zeit, Chancen, 07/2005, www.zeus.zeit.de/text/2005/07/C-Geb_9fhrenmythen, 05.05.2005

[15] O.V.-1.: Bologna-Prozess, in:http://de.wikipedia.org/wiki/Bologna-Prozess, 10.01.2006

[16] O.V.-2.: Studiengebühren: Finanzminister, ick hör dir trapsen, 12.02.2005, www.spiegel.de/unispiegel/geld/0,1518,druck-341276,00.html. 13.02.2005

[17] O.V.-3.: Interview zu Bezahlstudium: „Studiengebühren wirken abschreckend“, in: Spiegel Online, 28.01.2005, www.spiegel.de/unispiegel/geld/0,1518,druck338346,00.html, 13.02.2005

[18] O.V.-4.: Reform-Motor. Unis in der Offensive, in Focus-Online, www.bildung.focus.msn.de/bildung/bildung/uni-gebuehren?page=4\&drucken=1, 02.10 .2005

[19] O.V.-5.: Gebührenpläne in der Schublade, in Focus-Online, www.bildung.focus.msn.de/bildung/bildung/uni-gebuehren?interface=table\&id=1206, 02.10 .2005 
[20] O.V.-6.: Gebührenschraube. „Bummelstudenten“ müssen blechen, in Focus-Online, www.bildung.focus.msn.de/bildung/bildung/uni-gebuehren?page=2\&drucken=1, 02.10 .2005

[21] O.V.-7.: Experten bewerten Studiengebührfreiheit unterschiedlich, 17.04.2002, www.bundestag.de/bic/hib/2002/2002_099/04.html, 20.01.2005

[22] O.V.-8.: Gebühren für das Studium, 04.03.2005, www.bundestag.de/bic/hib/2005/0506/titelseite.01.html, 20.03.2005

[23] OV.-9.: Studiengebühren, in: Süddeutsche Zeitung, 7. September 2005

[24] O.V.-10.: Verfassungsklage. Bundesländer machen ernst, in Focus-Online, www.bildung.focus.msn.de/bildung/bildung/uni-gebuehren?page=3\&drucken=1, 02.10 .2005

[25] O.V.-11.: Nach BVG-Urteil. Start frei für Uni-Gebühren, in: Focus-Online, www.bildung.focus.msn.de/bildung/bildung/uni-gebuehren?page=1\&drucken=1, 02.10 .2005

[26] Rudolf, Nicole.: Studiengebühren: Ein alternatives Finanzierungsmodell für das deutsche Hochschulsystem? Seminararbeit im Studiengang Europäische Kultur und Wirtschaft, Sommersemester 2004, Ruhr-Universität Bochum

[27] Spiewak, Martin (a): Geist gegen Geld, in: Die Zeit, Wissen, 05/2005, www.zeus.zeit.de/text/2005/07/C-Geb_9fhren, 13.02.2005

[28] Spiewak, Martin (b): Kopf oder Zahl, in: Die Zeit, Politik, 05/2005, www.zeus.de/text/2005/05/01_2_Leiter, 13.02.2005

[29] Vereinte Dienstleistungsgewerkschaft (verdi): Erst bezahlen, dann lernen, Berlin 2005 